\title{
Preparation of Folic Acid-conjugated Albumin Nanoparticles Containing Paclitaxel using High- pressure Homogenisation Coagulation Method
}

\section{Yan Chu}

Qiqihar Medical College: Qiqihar Medical University

\section{Shuo Chai}

Qiqihar Medical College: Qiqihar Medical University

Hong Pan

Qiqihar Medical College: Qiqihar Medical University

Jiayi Qian

Qiqihar Medical College: Qiqihar Medical University

\section{Cuiyan Han}

Qiqihar Medical College: Qiqihar Medical University

Xiaoyu Sui ( $\nabla$ suixiaoyu@outlook.com )

Qiqihar Medical University https://orcid.org/0000-0001-8741-2902

Tingting Liu

Qiqihar Medical College: Qiqihar Medical University

\section{Nano Express}

Keywords: Albumin nanoparticles, Paclitaxel, Folic acid, High pressure homogenization, Cell uptake

Posted Date: March 9th, 2021

DOI: https://doi.org/10.21203/rs.3.rs-221888/v1

License: (c) (i) This work is licensed under a Creative Commons Attribution 4.0 International License.

Read Full License 
1

$7 \quad{ }^{*}$ Corresponding authors: Xiaoyu Sui, Tingting Liu, email: suixiaoyu@outlook.com

8 (Xiaoyu Sui) and ltting@outlook.com (Tingting Liu). 


\section{Abstract}

In this study, we prepared and evaluated folic acid-conjugated albumin-paclitaxel (FA-BSA-PTX) nanoparticles using a new green technique, called the high-pressure homogenisation coagulation method (HPHCM). The effect of process parameters such as BSA concentration, coagulant concentration, homogenisation time, homogenisation pressure, water/ethanol ratio, and BSA/PTX ratio was analysed to optimise nanoparticle size, albumin conversion rate, and encapsulation efficiency. BSA concentration was found to exert a great influence on albumin conversion rate and particle size. Meanwhile, the BSA/PTX ratio significantly affected the nanoparticle encapsulation efficiency. Electron microscopy showed that the freeze-dried particles mostly existed in the form of dimers and trimers with an average particle size of 300 $400 \mathrm{~nm}$. Infrared spectroscopy indicated that PTX was well encapsulated in BSA. Raman spectra of the synthesised nanoparticles indicated changes in the disulphide bond configuration and protein structure. In vitro drug-release analysis showed that crosslinked nanoparticles exhibited a sustained release. Furthermore, in vitro celluptake studies on HeLa cells showed that FA can be used as a targeting ligand for albumin carriers to enhance the active targeting effect of the nanoparticles with a high FR expression. These results suggest that HPHCM is an effective method to prepare FA-BSA-PTX drug-delivery systems.

Keywords: Albumin nanoparticles; Paclitaxel; Folic acid; High pressure homogenization; Cell uptake 


\section{Introduction}

Nanoparticle-based drug-delivery systems are considered to show great potential for cancer treatment as these carrier systems exhibit high drug absorption, adjustable drug-release rate, and targeted delivery, especially in the case of hydrophobic drugs $[1,2]$. Previous reports have suggested that nanoparticles hundreds of nanometers in diameter can passively reach tumors by virtue of the enhanced permeability and retention (EPR) effect [3]. Lately, nanoparticle drug-delivery systems with albumin as a carrier are receiving much attention. As an endogenous substance, albumin plays a unique role in reducing the phagocytosis of nanoparticles, thus prolonging the in vivo circulation of drugs [4,5]. In addition, albumin is a macromolecular carrier, whose pores can encapsulate hydrophobic compounds. Moreover, albumin can be tightly but reversibly bound to certain drugs through non-covalent bonds to realize drug transport in vivo and their release at the target cell surface [6]. Bovine serum albumin (BSA) is one of the most commonly used drug carrier proteins owing to its good biocompatibility, low cost, and easy purification [7]. BSA-based nanoparticle drug-delivery systems exhibit various functions and binding properties such as good stability and a high binding capacity for drugs with different physical and chemical properties. In addition, the BSA molecule contains functional groups such as amino and carboxyl groups owing to which albumin nanoparticle carrier surfaces can be easily modified by ligands $[8,9]$.

The main purpose of targeted cancer therapy is to deliver drugs to tumour cells in order to localise high drug concentrations at tumour sites and reduce side effects $[10,11]$. Passive targeting is inherent in drug-loaded nanoparticles. Meanwhile, to achieve an active targeting function, ligand modification may be adopted [12]. Folic acid (FA), a common targeting ligand, is a low molecular weight vitamin whose folate receptor (FR) is overexpressed on the surfaces of a variety of cancer cells such as breast, ovary, lung, kidney, colon, and brain cancer cells; however, its expression is minimal in healthy tissues and organs. The FR expression level in cancer cells is 100- 
300 times higher than that in normal tissues [13]. Therefore, targeted delivery can be achieved by FA-linked nanoparticles entering the cytoplasm via FR mediation and endocytosis. FR is extensively studied for cancer diagnosis and treatment [14] due to several key characteristics. Firstly, FA can be easily synthesised, is inexpensive, and is easy to chemically modify and characterise [15]. Secondly, FA itself has no immunogenicity and exhibits high structural stability even after chemical modification, thus maintaining a high affinity with FR [16].

Traditionally, albumin nanoparticles are prepared by chemical crosslinking, thermal denaturation, or desolvation. Chemical crosslinking is non-specific and depends only on the reactivity of the nucleophilic groups (such as amino and hydroxyl groups) in the protein structure. Nevertheless, the crosslinking agents used in this method, such as formaldehyde, exhibit high levels of toxicity and result in residues $[17,18]$. Thermal denaturation, by techniques such as solution thermal crosslinking or spray drying, irreversibly alters the protein structure and the resultant protein nanoparticles exhibit poor biodegradability and a wide size distribution [19]. Using the desolvation method, nanoparticles are obtained by continuously dropping a water-miscible organic phase, such as ethanol, into an aqueous albumin solution under continuous stirring until the solution becomes turbid [20]. The formed nanoparticles are morphologically unstable and require hardening by crosslinking with glutaraldehyde. During this process, however, bioactive macromolecules may be deactivated and residues of harmful solvents may be formed. Toxic solvents, such as acetone, chloroform, and dichloromethane are required in the methods described here, which may lead to adverse health effects [21].

Green chemistry is a powerful ally of the pharmaceutical industry [22]. To synthesise nanoparticles in a 'green' manner, a non-toxic solvent medium should be used; in addition, non-toxic materials should be used to stabilise these nanoparticles [23]. Most of the preparation methods reported thus far rely heavily on organic solvents, which leads to toxicity. To counter this problem, environmentally friendly and sustainable green strategies methods are also being developed to prepare 
nanoparticles [24,25].

In this study, we used a new green technique, called the high-pressure homogenisation coagulation method (HPHCM), to prepare albumin nanoparticles. In a water-based system, albumin nanoparticles were formed using a coagulant; simultaneously, superoxide dismutase, produced by high-pressure homogeneous cavitation, was used to promote the formation of disulphide bonds between the cysteine residues of protein molecules. FA-BSA-paclitaxel (PTX) nanoparticles targeting FR were prepared with PTX as the model drug. The size and entrapment efficiency of the nanoparticles were analysed along with their physical and chemical properties. The in vitro release characteristics of nanoparticles obtained by secondary curing with different crosslinking agents were compared. The uptake of FA-BSA-PTX into HeLa cells was evaluated using laser confocal microscopy (LCSM).

\section{Materials and methods}

\subsection{Materials}

PTX, calcium lactate, and gluconolactone were procured from Dalian Meilun Biology Technology Co., Ltd. (Dalian, China). BSA, calcium chloride, and magnesium chloride were purchased from Sigma Aldrich Co. Ltd. (Shanghai, China), and methanol was purchased from J\&K Scientific Ltd. (Beijing, China). N,N'Dicyclohexylcarbodiimide (DCC), FA, and N-hydroxysuccinimide (NHS) were obtained from Yuan Ye Biotechnology Co. Ltd. (Shanghai, China); ethanol and dichloromethane were purchased from Sinopharm Chemical Reagent Co. Ltd. (Beijing, China). Pure water was prepared using a Milli-Q water purification system (Millipore, Bedford, MA, USA).

\subsection{Preparation of albumin nanoparticles}

The schematic diagram of the experimental apparatus are shown in Fig. 1. An albumin solution was prepared by dissolving $20 \mathrm{mg}$ of the coagulant $\left(\mathrm{MgCl}_{2}\right)$ and 400 $\mathrm{mg}$ of BSA in $40 \mathrm{~mL}\left(50^{\circ} \mathrm{C}\right)$ of pure water. Meanwhile, $40 \mathrm{mg}$ of PTX was dissolved in ethanol. The albumin solution was slowly added to the material cup of a high-pressure 
homogeniser and the temperature of the circulating water bath was set at $50^{\circ} \mathrm{C}$. PTX solution was added dropwise $(1 \mathrm{~mL} / \mathrm{min})$ to this pre-heated albumin solution at a pressure of 150 bar. Subsequently, the homogenisation pressure was adjusted to the target value and after a set time period, drug-loaded albumin nanoparticle suspensions could be obtained. These solutions were centrifuged at $8000 \mathrm{rpm}$ for $8 \mathrm{~min}$ in an ultracentrifuge (CP70MX, Hitachi Koki Co. Ltd., Japan) and the supernatant was removed. Later, the obtained precipitates were lyophilised with cryoprotectant mannitol ( $1: 1$ mass ratio). After pre-freezing for $12 \mathrm{~h}$ at $-80^{\circ} \mathrm{C}$, the nanoparticles was freeze-dried at a shelf temperature of $-50{ }^{\circ} \mathrm{C}$ for $24 \mathrm{~h}$ at a chamber pressure of $1-20$ Pa in a lyophiliser (SJIA-10N, Ningbo Shuangjia Science Technology Development Co. Ltd., China).

\subsection{Preparation of crosslinked albumin nanoparticles}

An albumin-nanoparticle suspension was prepared as described in paragraph 2.2, centrifuged at $8000 \mathrm{rpm}$ for 8 min using an ultracentrifuge (CP70MX, Hitachi Koki Co. Ltd., Japan), and the supernatant was removed. Purified water $(6 \mathrm{~mL})$ was added to the reaction flask to disperse the precipitate. Three crosslinking agents (genipin, vanillin, and glutaraldehyde) were added to this dispersion and allowed to react for 90 $\min$ at $60{ }^{\circ} \mathrm{C}$. Subsequently, the precipitates were lyophilised with cryoprotectant mannitol (1:1 mass ratio). After prefreezing for $12 \mathrm{~h}$ at $-80^{\circ} \mathrm{C}$, freeze-drying was carried out at a shelf temperature of $-50{ }^{\circ} \mathrm{C}$ for $24 \mathrm{~h}$ at a chamber pressure of $1-20$ Pa in a lyophiliser (SJIA-10N, Ningbo Shuangjia Science Technology Development Co. Ltd., China).

\subsection{Preparation of FA-BSA-PTX and FITC-BSA-PTX nanoparticles}

FA-BSA-PTX nanoparticles were prepared through a reaction between FA-NHS and BSA-PTX [26]. FA-NHS was synthesised as follows. Initially, $300 \mathrm{mg}$ of FA was dissolved in $6 \mathrm{~mL}$ of dimethyl sulphoxide (DMSO) and to this solution, $150 \mu \mathrm{L}$ of triethylamine, $282 \mathrm{mg}$ of DCC, and $156 \mathrm{mg}$ of NHS were added. The resultant mixture was stirred overnight at room temperature $\left(25^{\circ} \mathrm{C}\right)$. The insoluble by-product, 
dicyclohexylure (DCU), was removed using filtration, and FA-NHS was precipitated by pouring the filtrate into an ice-cold solution of anhydrous ether containing $30 \%$ acetone. After centrifuging the precipitate and washing it with anhydrous ether, it was subjected to suction filtration and vacuum drying to yield FA-NHS.

A small quantity of FA-NHS $(10 \mathrm{mg})$ was dissolved in $2 \mathrm{~mL}$ of an $\mathrm{Na}_{2} \mathrm{CO}_{3} / \mathrm{NaHCO}_{3}$ buffer solution (0.2 M, pH 10); meanwhile, freeze-dried BSA-PTX nanoparticles were also dissolved in the same buffer solution. The prepared FA-NHS solution was added dropwise to the BSA-PTX solution and stirred gently for $2 \mathrm{~h}$. At the end of this time period, the FA-BSA-PTX solution was subjected to centrifugal ultrafiltration (50 kDa, $3,214 \times g, 10 \mathrm{~min}$ ) and washed with deionised water. The obtained product was then freeze-dried.

BSA was diluted in a $\mathrm{Na}_{2} \mathrm{CO}_{3} / \mathrm{NaHCO}_{3}$ buffer solution $(25 \mathrm{mM}, \mathrm{pH} 9.8)$ at a concentration of $5 \mathrm{mg} / \mathrm{mL}$. Subsequently, FITC was added to the BSA solution to yield a final concentration of $0.1 \mathrm{mg} / \mathrm{mL}$ and the solution was incubated overnight at room temperature. The final product was obtained by centrifuging the mixed solution in ultrafiltration tubes with a molecular weight cut-off of $50 \mathrm{kDa}$ at $12,857 \times \mathrm{g}$ for $10 \mathrm{~min}$. The product was washed with the $\mathrm{Na}_{2} \mathrm{CO}_{3} / \mathrm{NaHCO}_{3}$ buffer and centrifuged twice to obtain FITC-BSA. FITC-BSA-PTX and FITC-FA-BSA-PTX were prepared using a similar process.

2.5. Quantitative determination of PTX using high-performance liquid chromatography (HPLC)

PTX content was analysed on a C18 HPLC column $\left(250 \times 4.6 \mathrm{~mm}^{2}, 5 \mu \mathrm{m}\right.$; Shandong Lunan Ruihong Chemical Instrument Co. Ltd.) with methanol and water $(75: 25 \mathrm{v} / \mathrm{v})$ as mobile phase $A$ and methanol $(100 \%)$ as mobile phase $B(100 \%)$ at a flow rate of $1.0 \mathrm{~mL} / \mathrm{min}$. The analytes were evaluated using a wavelength detector at $227 \mathrm{~nm}$. The injection volume was set at $20 \mu \mathrm{L}$. A linear regression equation, $\mathrm{Y}=$ $0.6913 X+5.925\left(R^{2}=0.9995\right)$, where $Y$ indicates peak area and $X$ represents mass concentration, was observed in the range of $10-100 \mu \mathrm{g} / \mathrm{mL}$, illustrating a good linear 
relationship.

\subsection{Determination of PTX encapsulation efficiency}

The prepared BSA-PTX solution ( $320 \mu \mathrm{L})$ was pipetted into a centrifuge tube and to this solution, $2 \mathrm{~mL}$ of dichloromethane was added and vortexed for $5 \mathrm{~min}$; the solution was then left to equilibrate for $15 \mathrm{~min}$. The obtained solution was filtered through a $0.22 \mu \mathrm{m}$ syringe filter and transferred to a $1 \mathrm{~mL}$ penicillin bottle. The ultrafiltrate $(20 \mu \mathrm{L})$ was injected into the HPLC system and analysed at a UV absorption wavelength of $227 \mathrm{~nm}$; the content of PTX detected was called the content of free drug. Encapsulation efficiency (EE) was calculated according to the following equation

$$
E E(\%)=\frac{W_{\text {totat }}-W_{\text {free }}}{W_{\text {total }}}
$$

where $W_{\text {free }}$ is the amount of free drug and $W_{\text {total }}$ is the amount of total drug.

\subsection{Determination of the conversion rate of albumin}

The BSA-PTX solution $(4 \mathrm{~mL})$ was pipetted into a centrifuge tube and centrifuged at $10,000 \mathrm{rpm}$ for $30 \mathrm{~min}$ at $4{ }^{\circ} \mathrm{C}$. Subsequently, $50 \mu \mathrm{L}$ of the supernatant was pipetted out and added to $1950 \mu \mathrm{L}$ of purified water. The ultraviolet absorption of this solution was measured at $227 \mathrm{~nm}$ and the corresponding chromatogram was recorded. The recorded value represents absorbance by free albumin and was used to calculate the conversion of BSA-PTX nanoparticles. A linear regression equation, $Y=0.632 \mathrm{X}+$ $0.0245\left(R^{2}=0.9991\right)$ was obtained, where the absorbance $(Y)$ and albumin concentration $(X)$ exhibited a good linear relationship in the concentration range of 10 $100 \mu \mathrm{g} / \mathrm{mL}$. The albumin conversion rate(ACR) was calculated according to the following equation:

$$
A C R(\%)=\frac{W_{\text {total }}-W_{\text {free }}}{W_{\text {total }}}
$$

where $W_{\text {free }}$ is the amount of free albumin and $W_{\text {total }}$ is the amount of total albumin.

\subsection{Measurement of particle size of nanoparticles}


Albumin nanoparticles powders were dispersed with pure water and their mean size were characterised using a laser particle-size analyser (Nano ZS90 Malvern Instruments, UK).

\subsection{Fourier transform infrared (FTIR) spectroscopy}

The FTIR spectra of the samples were obtained on a Nicolet 6700 FTIR spectrometer (Thermo Scientific, Waltham, MA, USA). Each sample was mixed with potassium bromide in an agate mortar and compressed into a thin disc to form pelletised specimens. These specimens were scanned in the range of $4000-400 \mathrm{~cm}^{-}$ 1 at a resolution of $4 \mathrm{~cm}^{-1}$.

\subsection{Raman spectrometry (RS)}

FT-Raman spectra were acquired on a Nicolet 6700 FTIR with NXR FT-Raman module $(1064 \mathrm{~nm})$ in the wavenumber range of $4000-400 \mathrm{~cm}^{-1}$ over 64 scans with a $4 \mathrm{~cm}^{-1}$ spectral resolution.

2.11. Differential scanning calorimetry (DSC)

DSC measurements were conducted on an HSC-1 DSC scanning calorimeter (Hengjiu Instrument Ltd., Beijing, China). Samples (15 mg) were placed in aluminium pans, sealed using the sample pan press, and heated from 25 to $400{ }^{\circ} \mathrm{C}$ at a rate of $10^{\circ} \mathrm{C} / \mathrm{min}$ in a nitrogen atmosphere.

\subsection{X-ray diffraction (XRD)}

The structural properties of the samples were analysed using a D8 Focus X-Ray diffractometer (Bruker, Germany) with Cu-Ka radiation operating at $40 \mathrm{kV}$ and $40 \mathrm{~mA}$. The samples were scanned in the $2 \theta$ range of $3^{\circ}$ to $90^{\circ}$ at $0.02^{\circ} / \mathrm{min}$.

\subsection{Scanning electron microscopy (SEM)}

The samples were fixed on a SEM stub with a conductive carbon tape and sputtercoated with a thin layer of gold using an SBC-12 sputter-coater (KYKY Tech. Ltd., Beijing, China). The surface morphology of the samples was then observed using a 
S-4300 scanning electron microscope (Hitachi, Tokyo, Japan).

224

225

226

227

\subsection{Transmission electron microscopy (TEM)}

Nanoparticle shape and morphology were observed using a transmission electron microscope (Hitachi HT7700, Japan) operating at an accelerating voltage of $100 \mathrm{kV}$. The nanoparticle suspension was obtained by adding $5 \mathrm{mg}$ of nanoparticles powder to $5 \mathrm{~mL}$ of purified water at room temperature, vortexed for $10 \mathrm{~s}$, and equilibrated for $30 \mathrm{~s}$. From this solution, a drop was withdrawn with a micropipette and placed on a carbon-coated copper grid. Excess suspension was blotted from the grid with a filter paper. The remaining deposit was negatively stained with $1 \%$ phosphotungstic acid $(\mathrm{w} / \mathrm{w}, \mathrm{pH}$ 7.1). The excess was removed with a filter paper and the deposit was dried before analysis.

\subsection{In vitro drug release}

PTX release from the nanoparticles was analysed using the slurry method at (37 \pm 0.5) ${ }^{\circ} \mathrm{C}$. BSA-PTX nanoparticles were dissolved in $100 \mathrm{~mL}$ of a phosphate buffer solution (PBS, pH $7.4,37^{\circ} \mathrm{C}$ ) by stirring at $100 \mathrm{rpm}$. At predetermined time intervals, $2 \mathrm{~mL}$ of the release medium was removed and replaced with an equal amount of fresh release medium to maintain sink conditions, $2 \mathrm{~mL}$ alcohol was added, and filtered through a $0.45 \mu \mathrm{m}$ cellulose acetate membrane. The released drug content was analysed at $227 \mathrm{~nm}$ by HPLC (LC-1000D HPLC, Shandong Lunan Ruihong Chemical Instrument Co. Ltd.). Measurements were carried out in triplicate to calculate cumulative drug release.

\subsection{Cell uptake of the prepared nanoparticles}

To determine the specific affinity of PTX-BSA and FA-BSA-PTX towards FR, FITC was encapsulated in these two systems and LCSM was conducted to evaluate if the uptake of FA-BSA-PTX by HeLa cells was enhanced using FR. HeLa cells were plated at a density of $1.0 \times 10^{6} \mathrm{cells} /$ well and cultured overnight at $37^{\circ} \mathrm{C}$. Subsequently, the culture medium was discarded. Free FITC, FITC-BSA-PTX, and FITC-FA-BSA-PTX were diluted in the serum-free medium and each sample was added to two wells and 
251 incubated at $37^{\circ} \mathrm{C}$ for $3 \mathrm{~h}$. The cells were finally washed thrice with PBS, and analysed using LCSM to observe the uptake by HeLa cells.

253

254

255

256

257

258

259

260

261

262

263

264

265

266

267

268

269

270

271

272

273

\subsection{Data analysis}

Single correlation analysis was conducted to examine the effect of process parameters on the properties of albumin nanoparticles. All statistical evaluations were conducted with SPSS for Windows, version 17.0.0 (SPSS Inc., USA). A value of $p<$ 0.05 was considered to be statistically significant.

\section{Results and discussion}

\subsection{Coagulant screening}

According to the available literature, intermolecular functional regions can be generated by interactions between protein molecules and divalent metal ions to form precipitates and these precipitates enhance the stability and duration of protein interaction, resulting in a sustained release effect [27]. Moreover, the impact may be caused by the type of coagulant on the crosslinking mechanism and rate, thereby affecting the particle size and conversion rate of albumin nanoparticles. Taking these factors into account, in this study, the effect of different coagulants on the particle size and conversion rate was investigated.

As shown in Fig. 2, under the same preparation conditions, the conversion rate of the $50 \mathrm{mg}$ coagulant group $(1.25 \mathrm{mg} / \mathrm{mL})$ was higher than that of $20 \mathrm{mg}$ coagulant group $(0.5 \mathrm{mg} / \mathrm{mL})$. In terms of the conversion rate in the $0.5 \mathrm{mg} / \mathrm{mL}$ coagulant group, the following order was observed - magnesium chloride $>$ gluconolactone $>$ calcium chloride > calcium lactate, with magnesium chloride resulting in the highest conversion rate $(47 \%)$. In the case of the $1.25 \mathrm{mg} / \mathrm{mL}$ coagulant group, the observed order was magnesium chloride > gluconolactone > calcium chloride > calcium lactate, with magnesium chloride resulting in the highest conversion rate (84.5\%). Significant nanoparticle flocculation was observed when gluconolactone was used as the coagulant, owing to which the nanoparticles could not be effectively dispersed. 
The particle size of albumin nanoparticles in the $0.5 \mathrm{mg} / \mathrm{mL}$ coagulant group was smaller than that in the $1.25 \mathrm{mg} / \mathrm{mL}$ group. Particle-size order in the $0.5 \mathrm{mg} / \mathrm{mL}$ coagulant group was magnesium chloride < calcium chloride < calcium lactate < gluconolactone, with nanoparticles prepared using magnesium chloride being the smallest $(310.6 \mathrm{~nm})$. In the $1.25 \mathrm{mg} / \mathrm{mL}$ coagulant group, the particle-size order was magnesium chloride $<$ calcium chloride $<$ calcium lactate $<$ gluconolactone, with nanoparticles prepared using magnesium chloride being the smallest (398.6 nm).

Thus, considering the particle-size and conversion-rate indicators, magnesium chloride was selected as the optimal coagulant and used in the rest of the study.

\subsection{Effect of process parameters on the properties of albumin nanoparticles}

During the synthesis of albumin nanoparticles by HPHCM, nanoparticle size, albumin-conversion rate, and encapsulation rate can be adjusted by controlling the operating conditions such as BSA concentration, coagulant dosage, and homogenisation time. Therefore, we investigated the effect of different experimental conditions (Table 1) on the quality of the produced nanoparticles.

The effect of BSA concentration on the synthesised albumin nanoparticles is illustrated in Fig. 3a. As the BSA concentration increased from 5 to $40 \mathrm{mg} / \mathrm{mL}$, particle size gradually increased from 327 to $377 \mathrm{~nm}$. The change in particle size can be explained using the following relationships:

$$
J=A \exp ^{\left(-B /(\ln S)^{2}\right)}
$$
8

$$
A=N_{0} v
$$

\section{每}

301

$$
B=\frac{16 \pi \sigma^{3} V_{s}^{2}}{3 k^{3} T^{3}}
$$

$$
v=\frac{k T}{3 \pi a^{3} \eta}
$$


where $S$ is the degree of supersaturation, $J$ is the nucleation rate, $N_{0}$ is the initial number of molecules of solute per unit volume, $v$ is the frequency of molecular transport to the solid-liquid interface, Furthermore, $\mathrm{K}$ is the Boltzmann constant and $\mathrm{T}$ represents temperature. $\sigma$ indicates interfacial tension at the solid-liquid interface, and $V_{s}$ is the volume of solute molecules. $\eta$ is the viscosity of the surrounding solution, and $a$ is the mean effective diameter of the diffusing species.

Using Equation (3), it was found that viscosity increased as the BSA concentration increased. Larger particles were produced by slowing the nucleation due to a reduced protein transport between water and ethanol. In addition, higher protein concentrations led to greater supersaturation (Equation (4)), resulting in small nuclei. However, there is a greater chance of particle collision due to high supersaturation resulting in solidification, thus producing larger nanoparticles [28]. It has been reported by Prajapati et al. that coagulation occurs as BSA concentration increases, resulting in larger particles [29]. Albumin conversion initially increased and then decreased, with the highest conversion rate (45.8\%) observed at a BSA concentration of $10 \mathrm{mg} / \mathrm{mL}$. Shu et al. described that the conversion rate increased with an increase in BSA concentration in the range of $0.5-3 \mathrm{mg} / \mathrm{mL}$ [30]. In the current study, however, the low conversion rate observed at high concentrations may be attributed to the supersaturation effect, as described earlier. Meanwhile, the encapsulation efficiency varied from $85.3 \%$ to $90.8 \%$, with no obvious trend. Statistically, the influence of BSA concentration on particles size was highly significant $(p<0.01)$ and exerted a large influence on albumin-conversion rate $(p<0.05)$ into nanoparticles.

The effect of magnesium chloride concentration on the properties of albumin nanoparticles is illustrated in Fig. 3b. As the concentration of $\mathrm{MgCl}_{2}$ increased, nanoparticle size, conversion rate, and encapsulation rate increased gradually. An increase in the number of coagulant molecules resulted in an increase in the nucleation rate, which in turn increased the particle size and conversion rate.

The effect of water/ethanol volume ratio on albumin nanoparticles is shown in Fig. $3 c$. As the water/ethanol volume ratio decreased from 30 to 10 , the particle size initially 
decreased and then increased. The minimum particle size of $323 \mathrm{~nm}$ was observed at water/ethanol $=25 / 1$, which is consistent with previously reported results [31] . Meanwhile, the BSA conversion rate decreased when the water/ethanol volume ratio decreased from 30 to 10 . The encapsulation rate initially increased and then decreased and the highest value (92.2\%) was observed at water/ethanol $=20 / 1$.

The effect of homogenisation pressure on the properties of BSA nanoparticles is shown in Fig. 3d. As the pressure increased from 600 to 1000 bar, particle size initially decreased and then increased, with the smallest particles $(302 \mathrm{~nm})$ being obtained at 800 bar. It is well known that a homogeniser gradually warms up during operation and rapid reactant consumption occurs as the reaction rate increases at higher temperatures. As a result, the reactants are depleted, resulting in the formation of smaller nanoparticles at higher temperatures [32]. These results are consistent with those of Jenning et al. [33]. Later, the particle size increased gradually due to the exaggerated pressure of the homogeniser, an exorbitant instantaneous energy, and the solidification of particles [34]. In this process, the conversion rate also increased. Statistically, the influence of homogenisation pressure on conversion rate was highly significant $(p<0.01)$. The encapsulation rate initially increased and then decreased, reaching the highest value $(89.7 \%)$ at a homogenisation pressure of 700 bar.

The effect of homogenisation time on albumin nanoparticles is illustrated in Fig. 3e. When the homogenisation time increased from 3 to $9 \mathrm{~min}$, the particle size increased gradually while in the range of 9-15 min, it increased rapidly. This phenomenon can be attributed to an energy barrier between the particles. When individual particles approach each other, the attractive potential energy (EA) increases rapidly, whereas the repulsive potential energy (ER) changes more slowly. Generally speaking, there is a maximum value (Emax) in the total potential energy curve, which represents the energy barrier and prevents particles from absorbing each other. The Emax may be overcome when the particles approach each other. As the homogenisation time increases, more and more energy is accumulated. After $9 \mathrm{~min}$, the repulsive potential energy, Emax, could be overcome, owing to which individual nanoparticles were 
strongly attracted to each other, resulting in a sudden increase in particle size. Statistically, the influence of homogenisation time on particle size was found to be significant $(p<0.05)$. Furthermore, the conversion rate increased as the homogenisation time increased. Meanwhile, the encapsulation rate increased initially and then decreased, with the maximum value (91.4\%) observed at 12 min.

The effect of the BSA/PTX ratio on albumin nanoparticles is shown in Fig. 3f. When the BSA/PTX ratio decreased from 25 to 5 (dosage increases), the conversion rate and particle size increased; however, the encapsulation efficiency decreased. This indicates that the amount of BSA was not sufficient to encapsulate the entire loaded PTX amount. Similarly, when the dosage increased, the drug acted as a coagulant, resulting in many crystalline nuclei. At this time, BSA was adsorbed, resulting in particle adherence and large particles [35]. Statistically, the BSA/PTX ratio was found to significantly influence the encapsulation efficiency $(p<0.05)$.

Finally, the following reaction conditions were deemed to be optimal - BSA concentration $=10 \mathrm{mg} / \mathrm{mL}, \mathrm{MgCl}_{2}=0.5 \mathrm{mg} / \mathrm{mL}$, water/ethanol volume ratio $=20 / 1$, homogenisation pressure $=800$ bar, homogenisation time $=3 \mathrm{~min}$, and BSA $/ \mathrm{PTX}=$ $10 / 1$.

\subsection{Electron microscope analysis}

The morphology of BSA-PTX nanoparticles were analysed using TEM and SEM, as shown in Fig. 4. It can be seen in the SEM images (Fig. 4a) that albumin is in the form of irregular flakes while raw PTX is in the form of crystalline strips (Fig. 4b). It can also be concluded from the SEM images (Fig. 4c and d) of BSA-PTX nanoparticles that the freeze-dried particles were mostly in the form of dimers and trimers with an average particle size of $300 \mathrm{~nm}$; in terms of shape, most single particles were spherical. The TEM image of BSA-PTX nanoparticles (Fig. 4f) shows that the freeze-dried powder consisted of nearly spherical nanoparticles with an average particle size of $300 \mathrm{~nm}$. It can be seen in the partial image (Fig. 4e) that there are obvious black crystals in the middle of the particles; these are assumed to be cores formed by $\mathrm{MgCl}_{2}$ 
(coagulant). During the formation of albumin nanoparticles, the coagulant formed the core and albumin shells were formed around them. Faint PTX crystal strips could also be observed, implying that PTX was dispersed in the carrier in the form of crystals.

\subsection{FTIR analysis}

FTIR can be used to easily detect the relative intensity of amide bands in proteins, thus allowing us to analyse their structure. We employed FTIR to analyse the structure of albumin nanoparticles and investigate changes in their characteristic absorption peaks to determine their composition (Fig. 5). The IR spectrum of albumin included characteristic peaks at 1638, 1540, and $1238 \mathrm{~cm}^{-1}$, representing amide I (1600-1700 $\mathrm{cm}^{-1}, \mathrm{C}=\mathrm{O}$ stretching vibrations), amide II (1500-1600 $\mathrm{cm}^{-1}, \mathrm{~N}-\mathrm{H}$ in-plane bending and C-N stretching), and amide III (1220-1280 $\mathrm{cm}^{-1}, \mathrm{C}-\mathrm{N}$ stretching and $\mathrm{N}-\mathrm{H}$ in-plane bending) groups, respectively. The appearance of these peaks is mainly due to the fact that albumin is in a disordered coiled state [36]. It can be seen from the figure that the absorption band of amide I moved from 1638 to $1654 \mathrm{~cm}^{-1}$ after albumin was micronised, which may be due to the rearrangement of amino and carboxyl hydrogen bonds on the BSA peptide chain during the homogenisation process. Protein conformation undergoes changes once hydrogen bonds, which are the main forces responsible for the double helix structure in a protein, change [37]. No obvious changes could be observed in the FTIR spectrum of BSA, but the peak intensity corresponding to amide II $\left(1540 \mathrm{~cm}^{-1}\right)$ increased significantly, indicating that the C-N and/or $\mathrm{NH}$ bonds underwent changes due to interactions between different functional groups [38]. An obvious characteristic peak could be observed in the FTIR spectrum of $\mathrm{MgCl}_{2}$ at $2250 \mathrm{~cm}^{-1}$; however, it could not be observed in the spectra of magnesium chloride blank nanoparticles and magnesium chloride drug-loaded nanoparticles. This implies that the $\mathrm{MgCl}_{2}$ amount is so small that it has little effect on drug-loaded nanoparticles. The PTX spectrum included a ketocarbonyl $(C=O)$ stretching vibration peak at $1733 \mathrm{~cm}^{-1}$, which could also be observed in the spectrum of magnesium chloride drug-loaded nanoparticles. Thus, FTIR validated PTX encapsulation in albumin. 


\subsection{Raman spectroscopy analysis}

Raman spectroscopy is a rapid, simple, repeatable, and non-destructive qualitative and quantitative technique that does not require special sample preparation. Thus, we employed this method to analyse the BSA-based nanoparticles prepared in this study. As shown in Fig. 6, peaks were observed at 1655 and $1339 \mathrm{~cm}^{-1}$ in the spectrum corresponding to albumin, representing amide $\square$ and $\square$ groups [39]. After nanoparticle formation, the relative intensities of these peaks decreased. This phenomenon can be explained by the structural changes occurring during the formation of BSA nanoparticles [40]. S-S stretching vibrations were observed in the wavenumber range of $500-550 \mathrm{~cm}^{-1}$ and these can be used to evaluate the configuration characteristics of disulphide bonds. The Raman peak at $510 \mathrm{~cm}^{-1}$ is characteristic of the twist-twisttwist configuration. Upon nanoparticle formation, Raman peaks were observed at 525 $\mathrm{cm}^{-1}$ corresponding to twist-twist-trans and trans-twist-twist configurations, indicating changes in the disulphide bond configuration and protein structure.

3.7. Differential scanning calorimetry analysis

The DSC curves of BSA, magnesium chloride, magnesium chloride blank nanoparticles, magnesium chloride drug-loaded nanoparticles, and PTX are shown in $\sim 222{ }^{\circ} \mathrm{C}$; however, this peak shifted to $197^{\circ} \mathrm{C}$ and widened in the thermogram of magnesium chloride drug-loaded nanoparticles. Data analysis showed that albumin was denatured during nanoparticle formation, which is consistent with related reports [41]. PTX exhibited an endothermic peak at $\sim 219^{\circ} \mathrm{C}$, which corresponds to its melting point, indicating that the drug exists in a crystalline state. However, no characteristic endothermic peaks could be observed at this temperature in the thermogram of BSAPTX nanoparticles, indicating that the drug underwent phase transformation and converted into an amorphous structure (low drug contents might also be responsible for this observation). It is well known that polymers retard crystalline growth in drugs via surface adsorption. Thus, the disappearance of the PTX melting peak indicates that PTX crystallisation was inhibited by albumin during precipitation [42]. 


\subsection{X-ray diffraction analysis}

XRD was conducted to investigate structural changes in the drug and albumin during nanoparticle preparation (Fig. 8). Pure PTX exhibited a strong diffraction peak, indicating its crystalline nature. In contrast, BSA was present in an amorphous state, as described in other studies [43]. Multiple diffraction peaks can be seen in the XRD patterns of blank and drug-loaded nanoparticles, which is not the case in the XRD patterns of albumin nanoparticles obtained by different methods. This difference might be due to the binding of $\mathrm{MgCl}_{2}$ and albumin [44,45]. Furthermore, the original diffraction peaks of PTX could not be observed in the XRD patterns of the nanoparticles, suggesting that drug converted into an amorphous state [46].

\subsection{In vitro drug release}

The effect of different crosslinking agents (genipin, vanillin, and glutaraldehyde) on drug release from the nanoparticles in in vitro conditions was investigated [47,[48]. BSA-PTX nanoparticles were immersed in PBS $(\mathrm{pH} 7.4)$ at $37^{\circ} \mathrm{C}$ for $24 \mathrm{~h}$ in vitro and the amount of drug released was analysed (Fig. 9). While genipin $(20 \mathrm{mg})$ and vanillin $(13.45 \mathrm{mg})$ and glutaraldehyde $(1.6 \mathrm{~mL}, 0.1 \%)$ was added at a concentration of 4 $\mu \mathrm{L} / \mathrm{mg}$ albumin; the crosslinking time was set at $3 \mathrm{~h}$. A sustained release could be observed with crosslinked BSA-PTX nanoparticles, whereas non-crosslinked BSAPTX nanoparticles exhibited a rapid release, with a $37 \%$ initial release within $15 \mathrm{~min}$. The release rate reached $97 \%$ within $4 \mathrm{~h}$ after which it converted into a plateau. When the nanoparticles were in contact with the release medium, the drug adsorbed on nanoparticle surfaces was first dissolved and released, resulting in a burst-release effect. At $1 \mathrm{~h}$, the cumulative release rate was in the range of $42.61 \%$ to $59.32 \%$. Subsequently, the release rate increased to $82.92 \%$ to $91.70 \%$ within $6 \mathrm{~h}$, after which it gradually slowed down (plateau phase). The release mode was biphasic, i.e., a burst effect at the beginning followed by a slow release phase [49]. The release medium continuously penetrated the interior of the nanoparticles, resulting in the release of drug molecules embedded in their bulk. The dissolved drug diffused into the medium through carriers depending on the drug-concentration gradient inside and outside the 
477 particles; when accompanied by the slow dissolution of the carrier material, this resulted in a slow release. Such sustained release behaviour can be attributed to the decrease in the free volume of the matrix treated with the crosslinking agents and $\mathrm{MgCl}_{2}$, which may limit the diffusion of drugs into the protein network [27]. The observed release rate followed the trend, genipin group > glutaraldehyde group > vanillin group.

\subsection{Cell uptake of BSA-based nanoparticles}

The results of in vitro cell-uptake studies on BSA-based nanoparticles are illustrated in Fig. 10. Fluorescence could be clearly observed in the case of FITC-FABSA-PTX (Fig. 10A) nanoparticles when incubated with HeLa cells at $37^{\circ} \mathrm{C}$ for $3 \mathrm{~h}$, while FITC-BSA-PTX (Fig. 10B) exhibited a relatively weak fluorescence. In addition, fluorescence was absent in the case of FITC (Fig. 10C). It can be concluded from these observations that FA mediated the endocytosis of BSA-NP in HeLa cells with a high FR expression, which increased the number of nanoparticles entering the cells. In contrast, samples without folate coupling had no such function. The amount of folate-modified nanoparticles entering HeLa cells was significantly high. Therefore, it can be stated that FA can be used as a targeting ligand to enhance the active targeting effect of albumin nanoparticles on HeLa cells with a high FR expression.

\section{Conclusion}

In this study, a new green method, called HPHCM, was developed for the preparation of albumin nanoparticles. The effect of different coagulants on the particle size and conversion rate of albumin nanoparticles was evaluated and it was found that $\mathrm{MgCl}_{2}$ resulted in small particles and a high albumin-conversion rate. It was proved by single-factor experiments that BSA concentration, coagulant concentration, and homogenisation time influence the size of albumin nanoparticles, albumin-conversion rate, and encapsulation rate. SEM analysis indicated that the prepared albumin nanoparticles were spherical with an average size of $300 \mathrm{~nm}$ and easily formed dimers or trimers upon lyophilisation. DSC testing showed that albumin mainly existed in an 
505 amorphous form after nanoparticle formation. The peak shift corresponding to amide 506 I in the FTIR spectrum of the nanoparticles is evidence of changes in protein 507 conformation. Using Raman spectroscopy, these changes were ascribed to changes 508 in the disulphide bond configuration and protein structure. In vitro release studies 509 confirmed sustained release from crosslinked BSA-PTX nanoparticles for over $6 \mathrm{~h}$. In 510 terms of the crosslinking agent, the release rate followed the order genipin > 511 glutaraldehyde > vanillin. In vitro studies also showed that FA can be used as a 512 targeting ligand for albumin carriers to enhance the active targeting effect of BSA-PTX 513 nanoparticles on HeLa cells with a high FR expression. Finally, HPHCM is a simple 514 and feasible method with great potential to synthesise drug carriers. It should be noted 515 that BSA was used in this study with only four coagulants and three cross-linking 516 agents. Therefore, other kinds of albumin, coagulants, and cross-linking agents should 517 also be studied to develop efficient drug carriers for targeted delivery. 


\section{Abbreviations}

520

PTX: Paclitaxel; BSA: Bovine serum albumin; FA: Folic acid; HPHCM: high-pressure homogenisation coagulation method; DSC: Differential scanning calorimetry; XRD: X-ray diffraction; FTIR: Fourier transform infrared spectroscopy; TEM: Transmission electron microscopy; EE: Encapsulation efficiency; ACR: Albumin conversion rate

\section{Acknowledgements}

The authors gratefully acknowledge the financial supports of the National Natural

526 Science Foundation of China (Grant No. 21908119) and the Natural Science

527 Foundation of Heilongjiang Province of China (Grant No. H2016097).

\section{Authors' Contributions}

529 CY, LT, SX participated in the design of the study. CY, LT, SX, CS, PH, QJ and HC 530 performed the experiments and materials characterization. CY, LT and SX drafted 531 the manuscript. All authors read and approved the final manuscript.

\section{Authors' Information}

533 All authors (Dr. Xiaoyu Sui, Dr. Tingting Liu, Dr. Cuiyan Han, Yan Chu, Shuo Chai, Hong Pan and Jiayi Qian) are from Qiqihar Medical University, China.

\section{Competing Interests}

536 The authors declare that they have no competing interests. 


\section{References}

1. Bansal A, Kapoor DN, Kapil R, et al (2011) Design and development of paclitaxel-loaded bovine serum albumin nanoparticles for brain targeting. Acta Pharm 61:141-156.

2. Dang Y, Guan J (2020) Nanoparticle-based drug delivery systems for cancer therapy. Smart Mater Med 1:10-19.

3. Thao LQ, Byeon HJ, Lee C, et al (2016) Doxorubicin-Bound Albumin Nanoparticles Containing a TRAIL Protein for Targeted Treatment of Colon Cancer. Pharm Res 33:615-626.

4. Karami E, Behdani M, Kazemi-Lomedasht F (2020) Albumin nanoparticles as nanocarriers for drug delivery: Focusing on antibody and nanobody delivery and albumin-based drugs. J Drug Deliv Sci Technol 55:101471.

5. Bora DK, Deb P (2009) Fatty acid binding domain mediated conjugation of ultrafine magnetic nanoparticles with albumin protein. Nanoscale Res Lett 4:138-143.

6. Kratz F (2008) Albumin as a drug carrier: Design of prodrugs, drug conjugates and nanoparticles. J Control Release 132:171-183.

7. Zu Y, Meng L, Zhao X, et al (2013) Preparation of 10-hydroxycamptothecinloaded glycyrrhizic acid-conjugated bovine serum albumin nanoparticles for hepatocellular carcinoma-targeted drug delivery. Int J Nanomedicine 8:12071222.

8. Yewale C, Baradia D, Vhora I, Misra A (2013) Proteins: Emerging carrier for delivery of cancer therapeutics. Expert Opin Drug Deliv 10:1429-1448.

9. Gong $\mathrm{T}$, Tan $\mathrm{T}$, Zhang $\mathrm{P}$, et al (2020) Palmitic acid-modified bovine serum albumin nanoparticles target scavenger receptor-A on activated macrophages to treat rheumatoid arthritis. Biomaterials 258:120296. 
10. Kudarha RR, Sawant KK (2017) Albumin based versatile multifunctional nanocarriers for cancer therapy: Fabrication, surface modification, multimodal therapeutics and imaging approaches. Mater Sci Eng C 81:607-626.

11. Zhao D, Zhao X, Zu Y, et al (2010) Preparation, characterization, and in vitro targeted delivery of folate-decorated paclitaxel-loaded bovine serum albumin nanoparticles. Int J Nanomedicine 5:669-677.

12. Ling D, Hackett MJ, Hyeon T (2014) Surface ligands in synthesis, modification, assembly and biomedical applications of nanoparticles. Nano Today 9:457477.

13. Nosrati H, Abbasi R, Charmi J, et al (2018) Folic acid conjugated bovine serum albumin: An efficient smart and tumor targeted biomacromolecule for inhibition folate receptor positive cancer cells. Int J Biol Macromol 117:1125-1132.

14. Akbarian A, Ebtekar M, Pakravan N, Hassan ZM (2020) Folate receptor alpha targeted delivery of artemether to breast cancer cells with folate-decorated human serum albumin nanoparticles. Int J Biol Macromol 152:90-101.

15. Qi L, Guo Y, Luan J, et al (2014) Folate-modified bexarotene-loaded bovine serum albumin nanoparticles as a promising tumor-targeting delivery system. $\mathrm{J}$ Mater Chem B 2:8361-8371.

16. Zhang L, Hou S, Mao S, et al (2004) Uptake of folate-conjugated albumin nanoparticles to the SKOV3 cells. Int J Pharm 287:155-162.

17. Steinhauser IM, Langer K, Strebhardt KM, Spänkuch B (2008) Effect of trastuzumab-modified antisense oligonucleotide-loaded human serum albumin nanoparticles prepared by heat denaturation. Biomaterials 29:4022-4028.

18. Yang R, Zheng $Y$, Wang Q, Zhao L (2018) Curcumin-loaded chitosan-bovine serum albumin nanoparticles potentially enhanced $A \beta 42$ phagocytosis and modulated macrophage polarization in Alzheimer's disease. Nanoscale Res Lett 13:1-9. 
19. Müller BG, Leuenberger H, Kissel T (1996) Albumin nanospheres as carriers for passive drug targeting: An optimized manufacturing technique. Pharm. Res. 13:32-37

20. Jahanban-Esfahlan A, Dastmalchi S, Davaran S (2016) A simple improved desolvation method for the rapid preparation of albumin nanoparticles. Int $\mathrm{J}$ Biol Macromol 91:703-709.

21. Langer K, Balthasar S, Vogel V, et al (2003) Optimization of the preparation process for human serum albumin (HSA) nanoparticles. Int J Pharm 257:169180.

22. Koenig SG, Dillon B (2017) Driving toward greener chemistry in the pharmaceutical industry. Curr Opin Green Sustain Chem 7:56-59.

23. Sharma RK, Gulati S, Mehta S (2012) Preparation of gold nanoparticles using tea: A green chemistry experiment. J Chem Educ 89:1316-1318.

24. Karthika S, Radhakrishnan TK, Kalaichelvi P (2020) Crystallization and Kinetic Studies of an Active Pharmaceutical Compound Using Ethyl Lactate As a Green Solvent. ACS Sustain Chem Eng 8:1527-1537.

25. Yew YP, Shameli K, Miyake M, et al (2016) Green Synthesis of Magnetite (Fe3O4) Nanoparticles Using Seaweed (Kappaphycus alvarezii) Extract. Nanoscale Res Lett 11:012-014.

26. Sun $Y$, Zhao Y, Teng S, et al (2019) Folic acid receptor-targeted human serum albumin nanoparticle formulation of cabazitaxel for tumor therapy. Int $\mathrm{J}$ Nanomedicine 14:135-148.

27. Wang W, Lei $Y$, Sui $\mathrm{H}$, et al (2017) Fabrication and evaluation of nanoparticleassembled BSA microparticles for enhanced liver delivery of glycyrrhetinic acid. Artif Cells, Nanomedicine Biotechnol 45:740-747.

28. Galisteo-González F, Molina-Bolívar JA (2014) Systematic study on the 
preparation of BSA nanoparticles. Colloids Surfaces B Biointerfaces 123:286292.

29. Prajapati A, Srivastava A (2019) Preparation, Characterization and Encapsulation Efficiency of Egg Albumin Nanoparticles Using EDC as Crosslinker. J Sci Ind Res 78:703-705

30. Shu S, Zhang X, Teng D, et al (2009) Polyelectrolyte nanoparticles based on water-soluble chitosan-poly(l-aspartic acid)-polyethylene glycol for controlled protein release. Carbohydr Res 344:1197-1204.

31. Dang F, Enomoto N, Hojo J, Enpuku K (2009) Sonochemical synthesis of monodispersed magnetite nanoparticles by using an ethanol-water mixed solvent. Ultrason Sonochem 16:649-654.

32. Mohammed Fayaz A, Balaji K, Kalaichelvan PT, Venkatesan R (2009) Fungal based synthesis of silver nanoparticles-An effect of temperature on the size of particles. Colloids Surfaces B Biointerfaces 74:123-126.

33. Jenning V, Lippacher A, Gohla SH (2002) Medium scale production of solid lipid nanoparticles (SLN) by high pressure homogenization. J Microencapsul 19:1-10.

34. Yazdani F, Edrissi M (2010) Effect of pressure on the size of magnetite nanoparticles in the coprecipitation synthesis. Mater Sci Eng B Solid-State Mater Adv Technol 171:86-89.

35. Yang L, Cui F, Cun D, et al (2007) Preparation, characterization and biodistribution of the lactone form of 10-hydroxycamptothecin (HCPT)-loaded bovine serum albumin (BSA) nanoparticles. Int J Pharm 340:163-172.

36. Tang J, Luan F, Chen X (2006) Binding analysis of glycyrrhetinic acid to human serum albumin: Fluorescence spectroscopy, FTIR, and molecular modeling. Bioorganic Med Chem 14:3210-3217. 
37. Shi XJ, Li D, Xie J, et al (2012) Spectroscopic investigation of the interactions between gold nanoparticles and bovine serum albumin. Chinese Sci Bull 57:1109-1115.

38. Costa BC, Ximenes VF, Lisboa-filho PN (2017) Synthetic nanoparticles of bovine serum albumin with entrapped salicylic acid. 11-21

39. Bhogale A, Patel N, Mariam J, et al (2014) Colloids and Surfaces B : Biointerfaces Comprehensive studies on the interaction of copper nanoparticles with bovine serum albumin using various spectroscopies. Colloids Surfaces B Biointerfaces 113:276-284.

40. Rohiwal SS, Satvekar RK, Tiwari AP, et al (2015) Investigating the influence of effective parameters on molecular characteristics of bovine serum albumin nanoparticles. Appl Surf Sci 334:157-164.

41. Dinarvand R, Taheri A, Atyabi F, et al (2011) Nanoparticles of conjugated methotrexate-human serum albumin: Preparation and cytotoxicity evaluations. J Nanomater 2011:5.

42. Honary S, Jahanshahi M, Golbayani R, et al (2010) Doxorubicin-loaded albumin nanoparticles: Formulation and characterization. J Nanosci Nanotechnol 10:7752-7757.

43. Ruttala HB, Ramasamy T, Shin BS, et al (2017) Layer-by-Layer Assembly of Hierarchical Nanoarchitectures to Enhance the Systemic Performance of Nanoparticle Albumin-bound Paclitaxel. Elsevier B.V.

44. Wang D, Liang N, Kawashima Y, et al (2019) Biotin-modified bovine serum albumin nanoparticles as a potential drug delivery system for paclitaxel. $\mathrm{J}$ Mater Sci 54:8613-8626.

45. Zhao Y, Cai C, Liu M, et al (2020) Drug-binding albumins forming stabilized nanoparticles for co-delivery of paclitaxel and resveratrol: In vitro/in vivo evaluation and binding properties investigation. Int J Biol Macromol 153:873- 
882.

46. Ruttala HB, Ko YT (2015) Liposome encapsulated albumin-paclitaxel nanoparticle for enhanced antitumor efficacy. Pharm Res 32:1002-1016.

47. Elzoghby AO, Samy WM, Elgindy NA (2012) Albumin-based nanoparticles as potential controlled release drug delivery systems. J Control Release 157:168182.

48. Song F, Zhang LM, Yang C, Yan L (2009) Genipin-crosslinked casein hydrogels for controlled drug delivery. Int J Pharm 373:41-47.

49. Sahin S, Selek H, Ponchel G, et al (2002) Preparation, characterization and in vivo distribution of terbutaline sulfate loaded albumin microspheres. J Control Release 82:345-358. 
682

683

684

685

686

687

688

689

690

691

692

693

694

695

696

697

698

699

700

701

702

703

704

\section{Figure legends}

Fig. 1. Schematic diagram of the experimental apparatus.(1), High pressure homogenizer; (2), Material cup; (3), Drug solution; (4), Peristaltic pump; (5), Heating circulator bath.

Fig. 2. Influence of coagulant concentration and types of coagulants on the mean particle size and BSA conversion efficiency.

Fig. 3. Influence of (a) BSA concentration ( $\mathrm{mg} / \mathrm{mL})$, (b) $\mathrm{MgCl}_{2}$ concentration $(\mathrm{mg} / \mathrm{mL})$, (c) The volume ratio of water and ethanol, (d) Homogeneous pressure (bar), (e) Homogeneous time ( $\mathrm{min}$ ) and (f) Mass ratio of BSA and PTX on the mean particle size, encapsulation efficiency and total conversion.

Fig. 4. Scanning electron micrographs of BSA(a), PTX(b), BSA-PTX(c) and (d), Transmission Electron Microscopy of BSA-PTX (e) and (f).

Fig. 5. FTIR spectra of $\mathrm{BSA}$; $\mathrm{MgCl}_{2}$; NP; PTX-BSA; and PTX. The characteristic vibration bands related to BSA and PTX can be seen in the PTX-BSA NPS nanoparticles.

Fig. 6. The DSC thermograms of BSA; $\mathrm{MgCl}_{2}$; NP; PTX-BSA; and PTX .

Fig. 7. The XRD spectra of $\mathrm{BSA}$; $\mathrm{MgCl}_{2}$; NP; PTX-BSA; and PTX.

Fig. 8. Raman spectrum of BSA; $\mathrm{MgCl}_{2}$; NP; PTX-BSA; and PTX.

Fig. 9. In vitro release profiles of PTX from PTX-BSA. Crosslinking agent dosage, glutaraldehyde/BSA $0.4 \mu \mathrm{l} / \mathrm{mg}$; genipin/BSA $0.05 \mathrm{mg} / \mathrm{mg}$; vanillin/BSA $0.02 \mathrm{mg} / \mathrm{mg}$.

Fig.10. Laser scanning confocal microscope images of HeLa cells after incubation with PTX-BSA. The LSCM images of the FITC-FA-BSA-PTX (A), FITC-BSA-PTX (B) and FITC (C). 

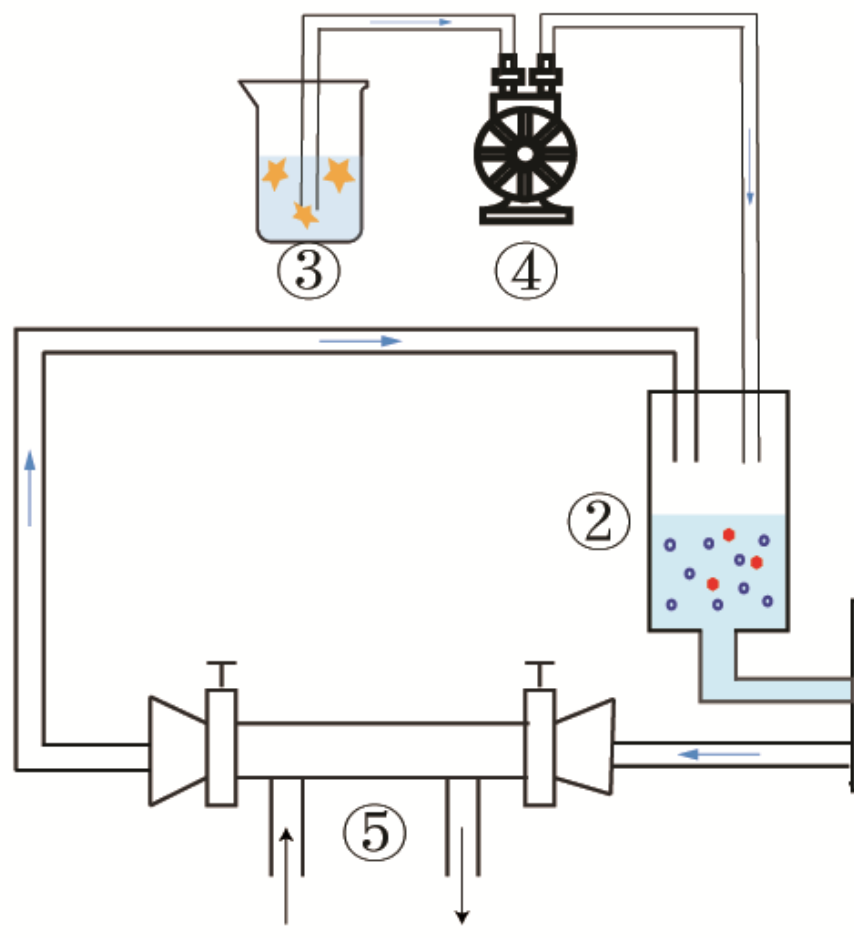

Heating circulator bath

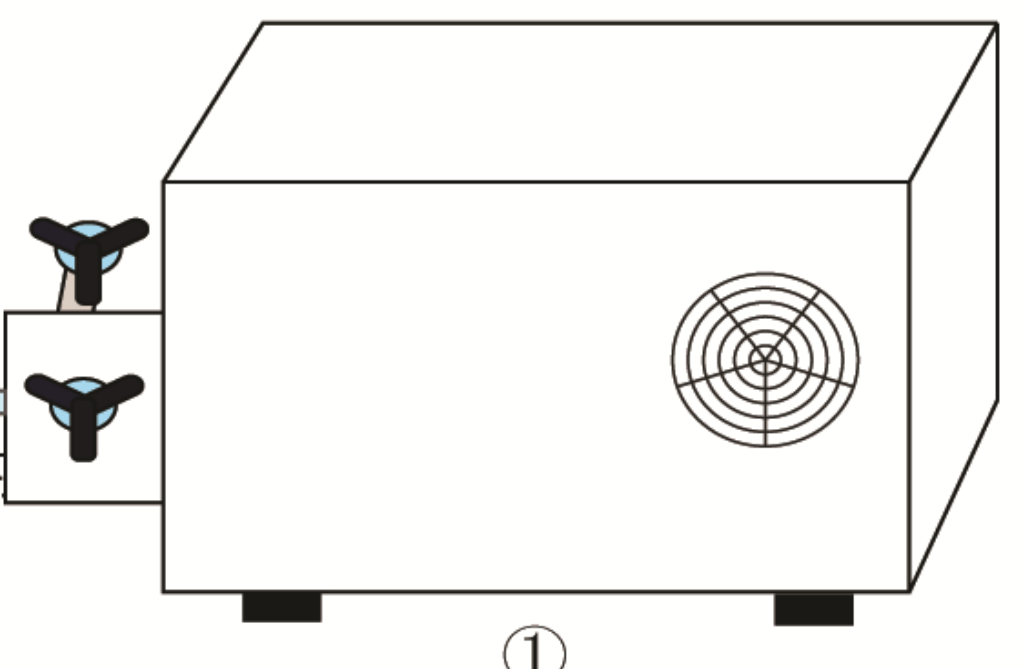

Figure 1

Schematic diagram of the experimental apparatus. $\otimes$, High pressure homogenizer; $\nabla$, Material cup; $\nabla$, Drug solution; 囚, Peristaltic pump; 囚, Heating circulator bath.
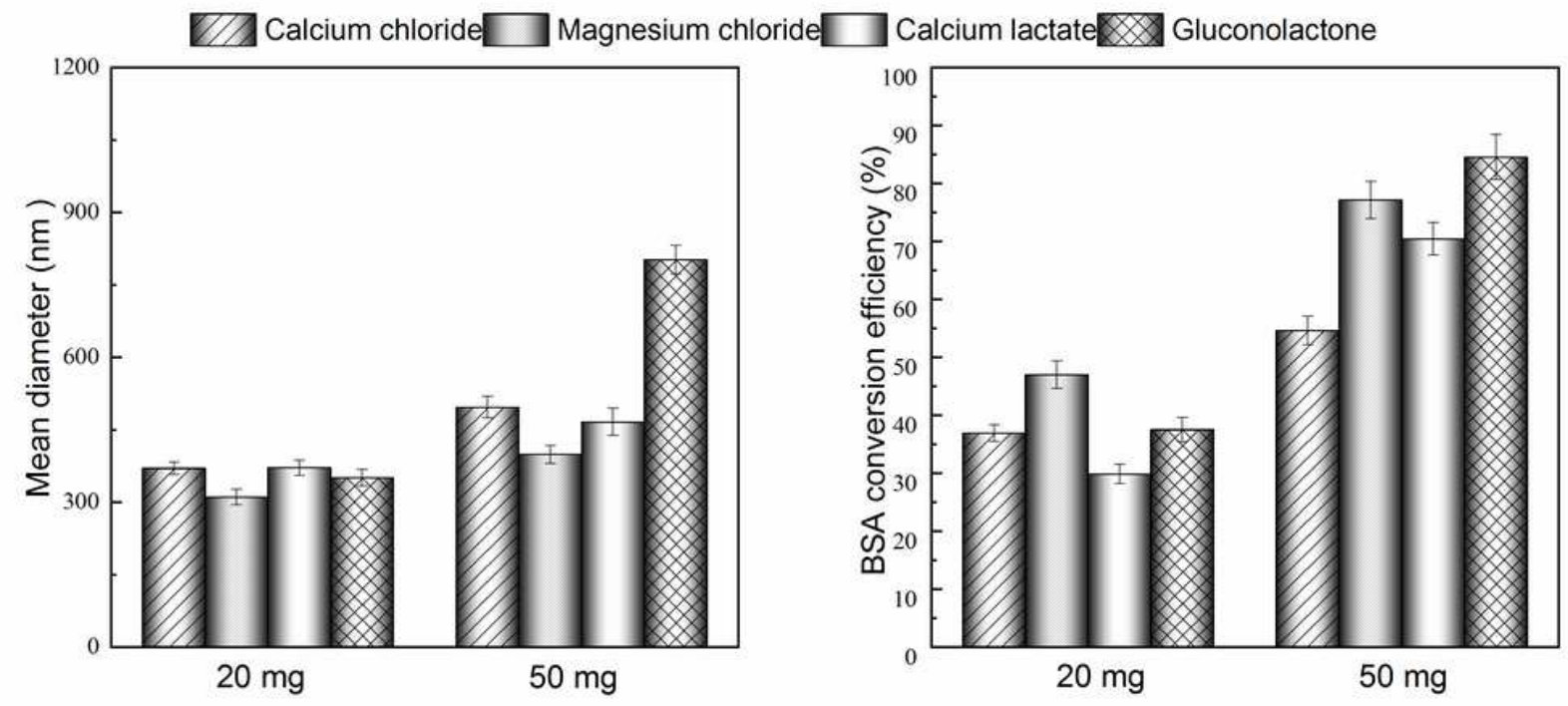
Figure 2

Influence of coagulant concentration and types of coagulants on the mean particle size and BSA conversion efficiency.
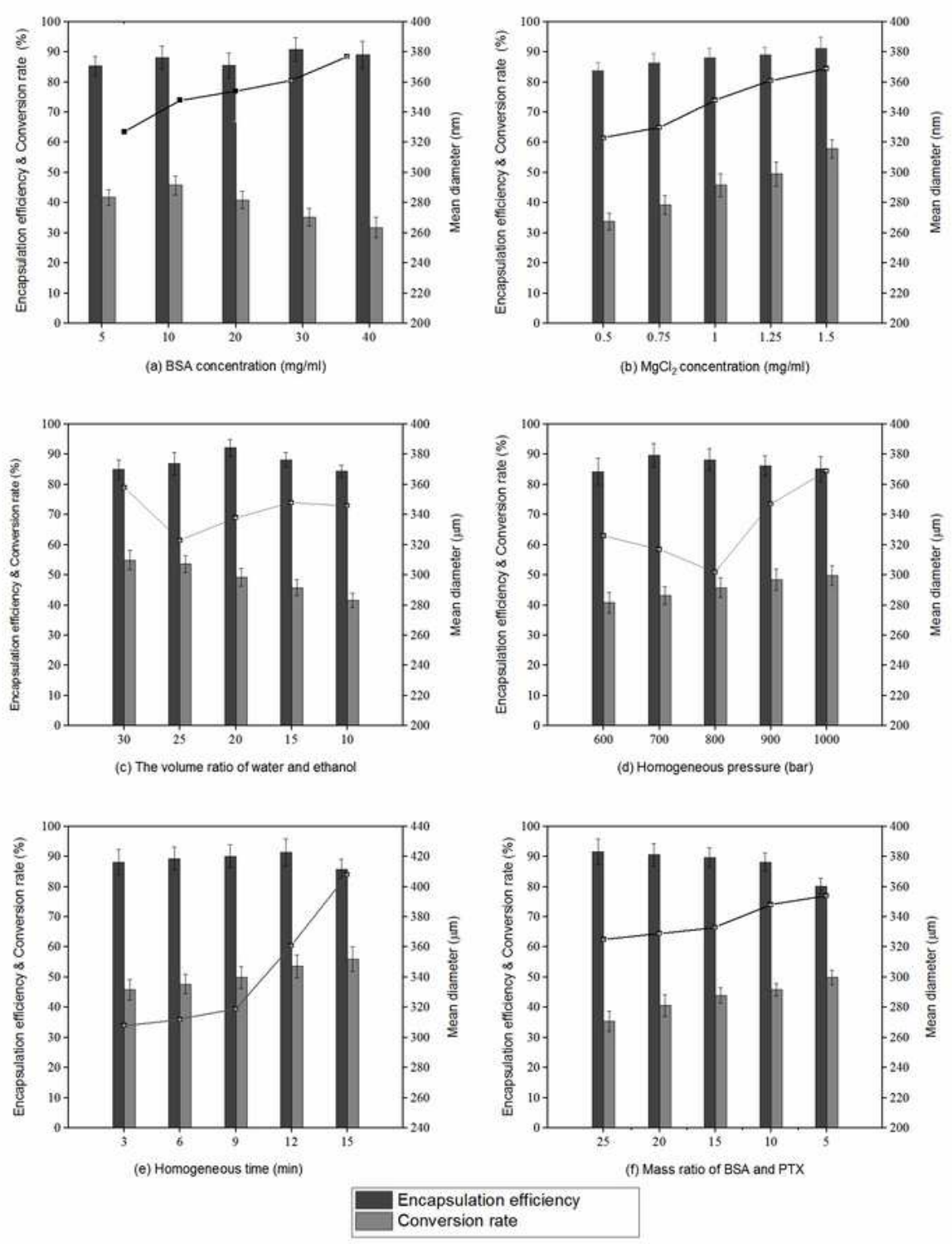

Figure 3 
Influence of (a) BSA concentration ( $\mathrm{mg} / \mathrm{mL}$ ), (b) $\mathrm{MgCl} 2$ concentration $(\mathrm{mg} / \mathrm{mL})$, (c) The volume ratio of water and ethanol, (d) Homogeneous pressure (bar), (e) Homogeneous time (min) and (f) Mass ratio of BSA and PTX on the mean particle size, encapsulation efficiency and total conversion.
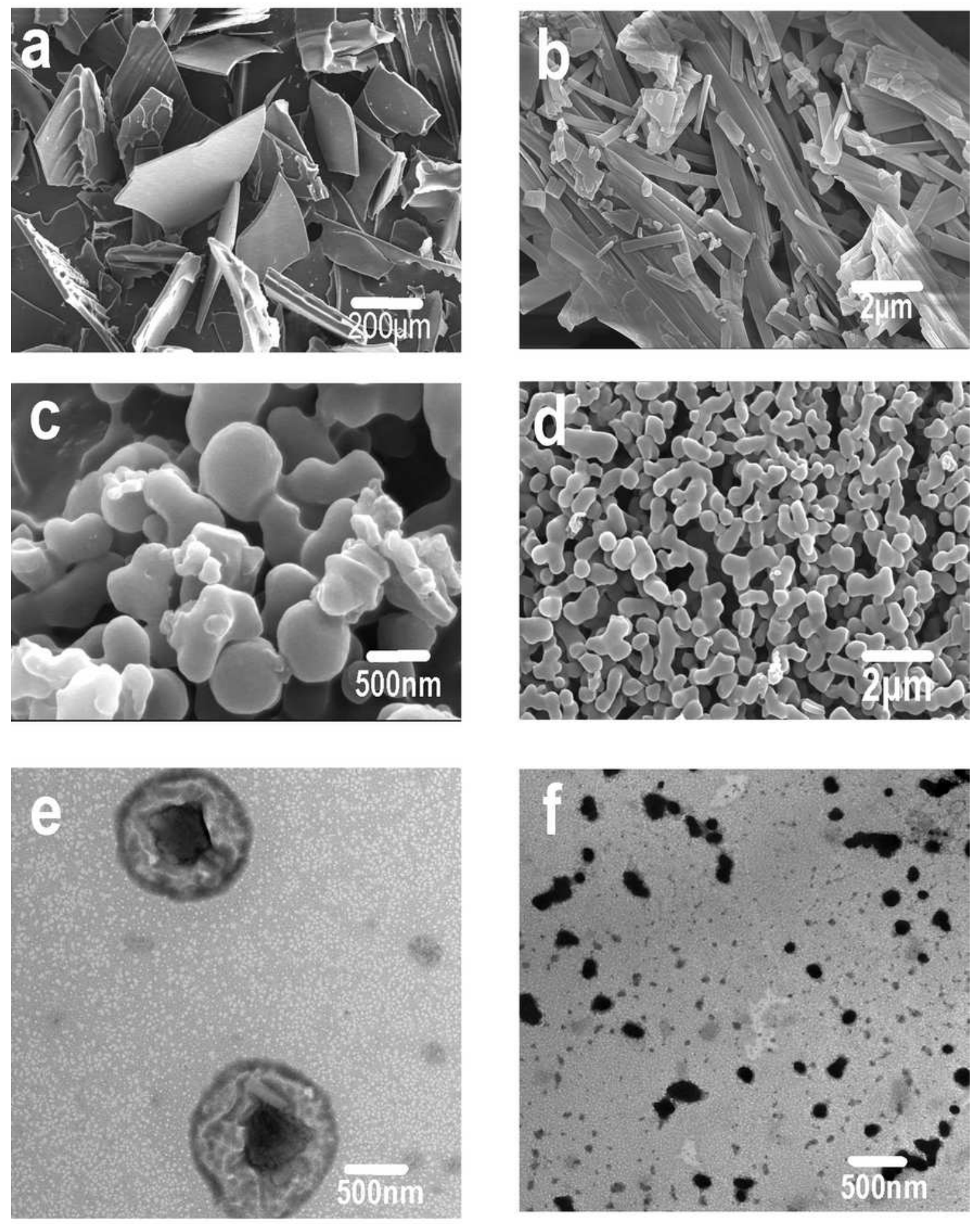

Figure 4

Scanning electron micrographs of BSA(a), PTX(b), BSA-PTX(c) and (d), Transmission Electron Microscopy of BSA-PTX (e) and (f). 


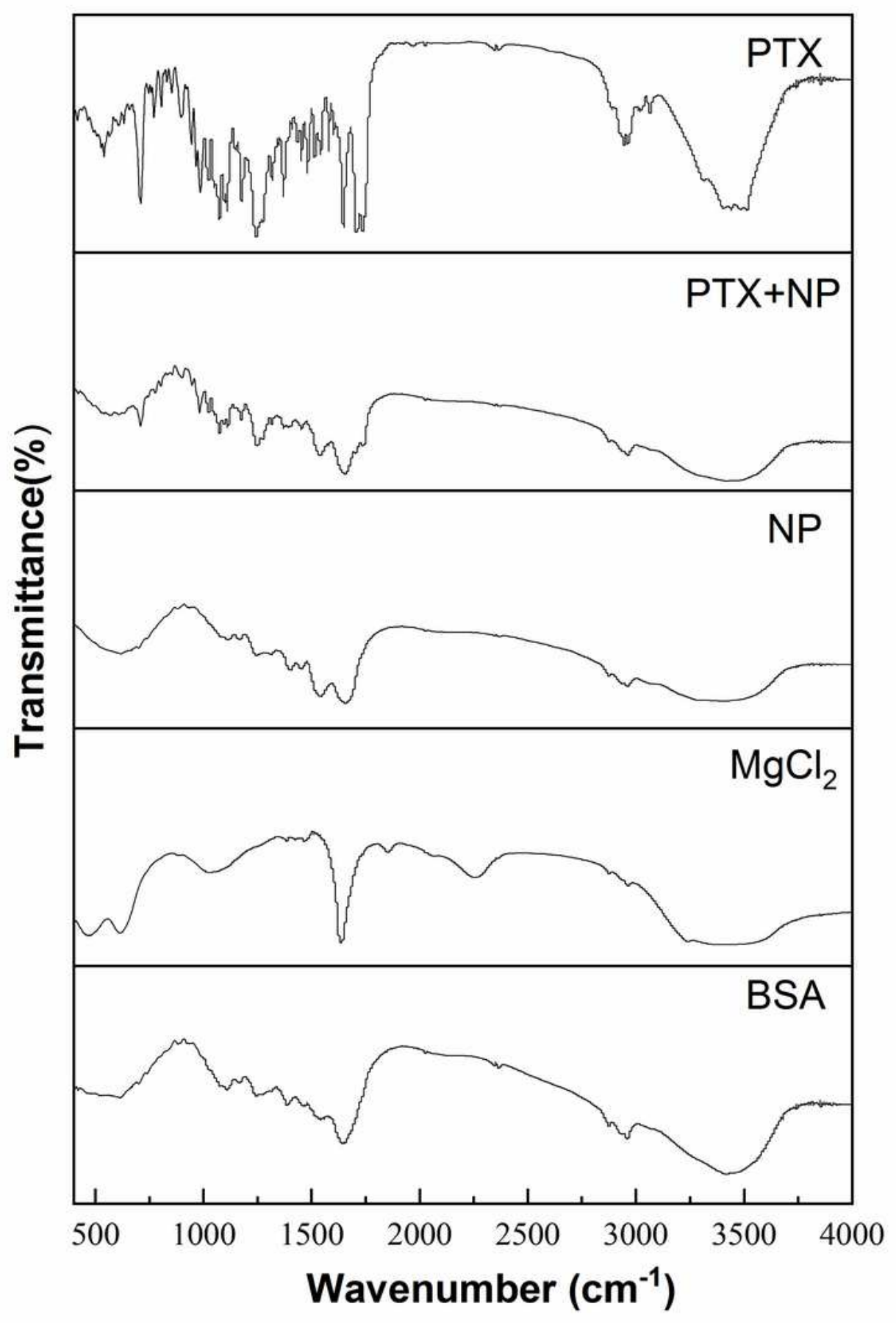

Figure 5

FTIR spectra of BSA; MgCl2; NP; PTX-BSA; and PTX. The characteristic vibration bands related to BSA and PTX can be seen in the PTX-BSA NPs nanoparticles. 


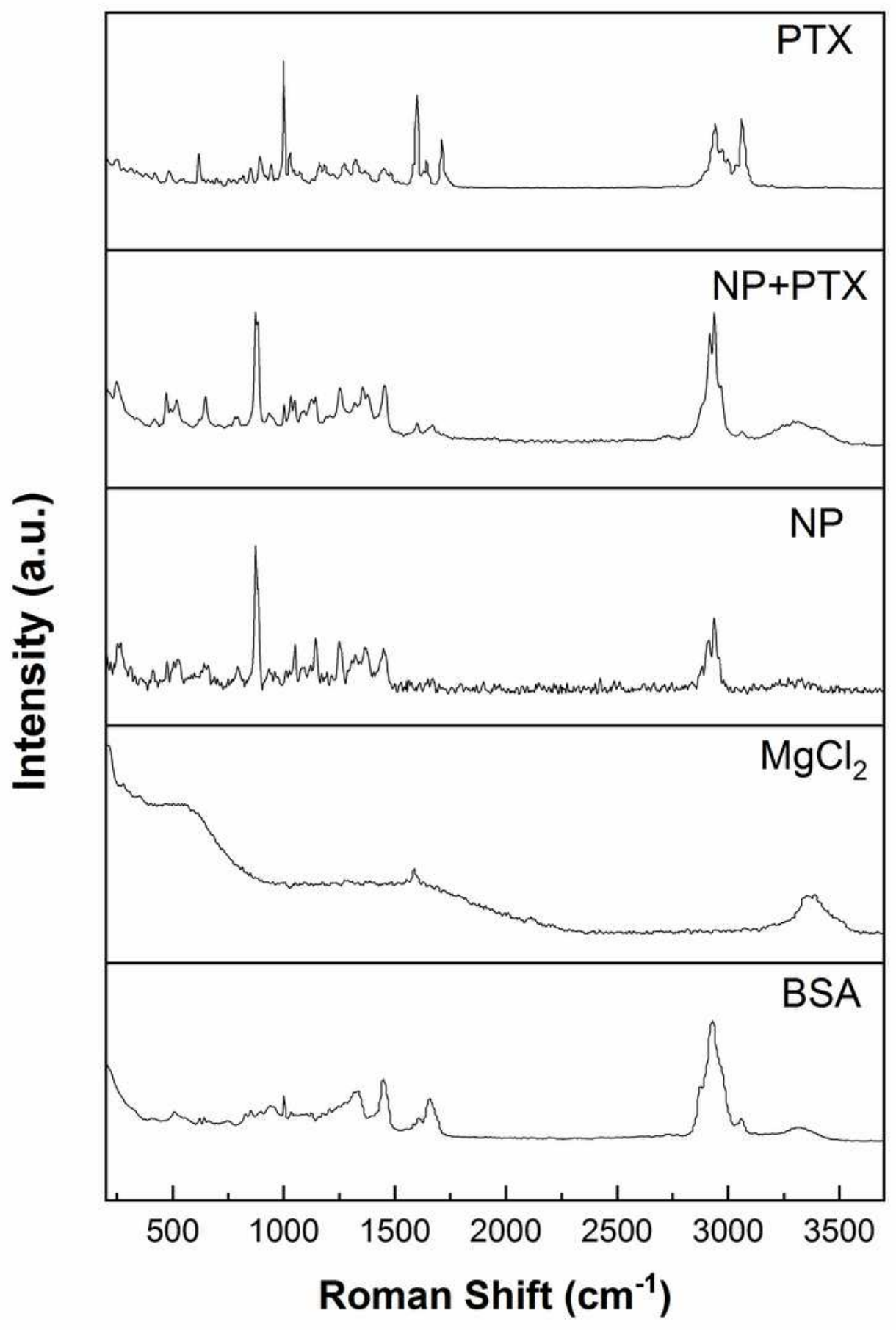

Figure 6

The DSC thermograms of BSA; MgCl2; NP; PTX-BSA; and PTX. 


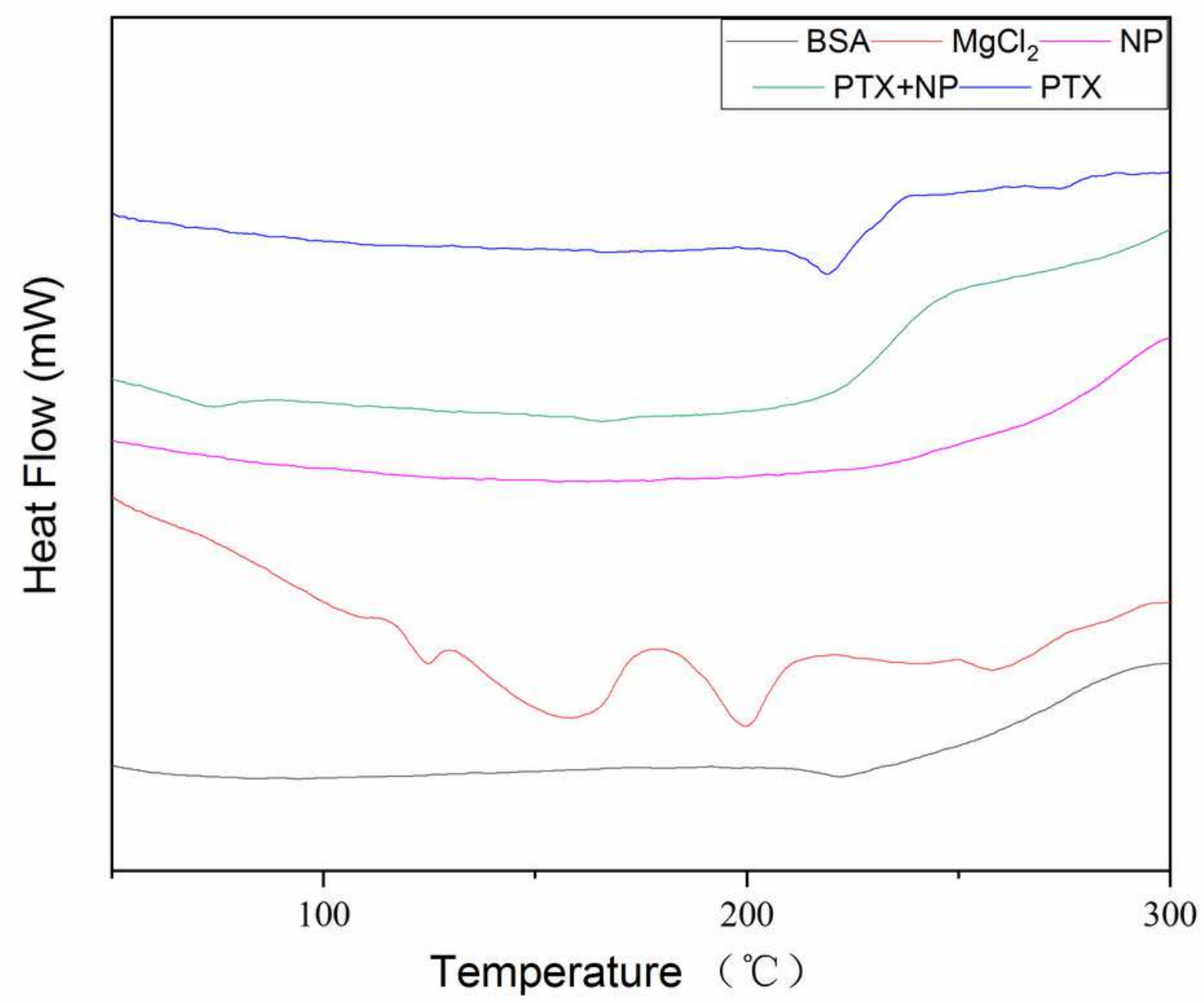

Figure 7

The XRD spectra of BSA ; MgCl2; NP; PTX-BSA; and PTX . 


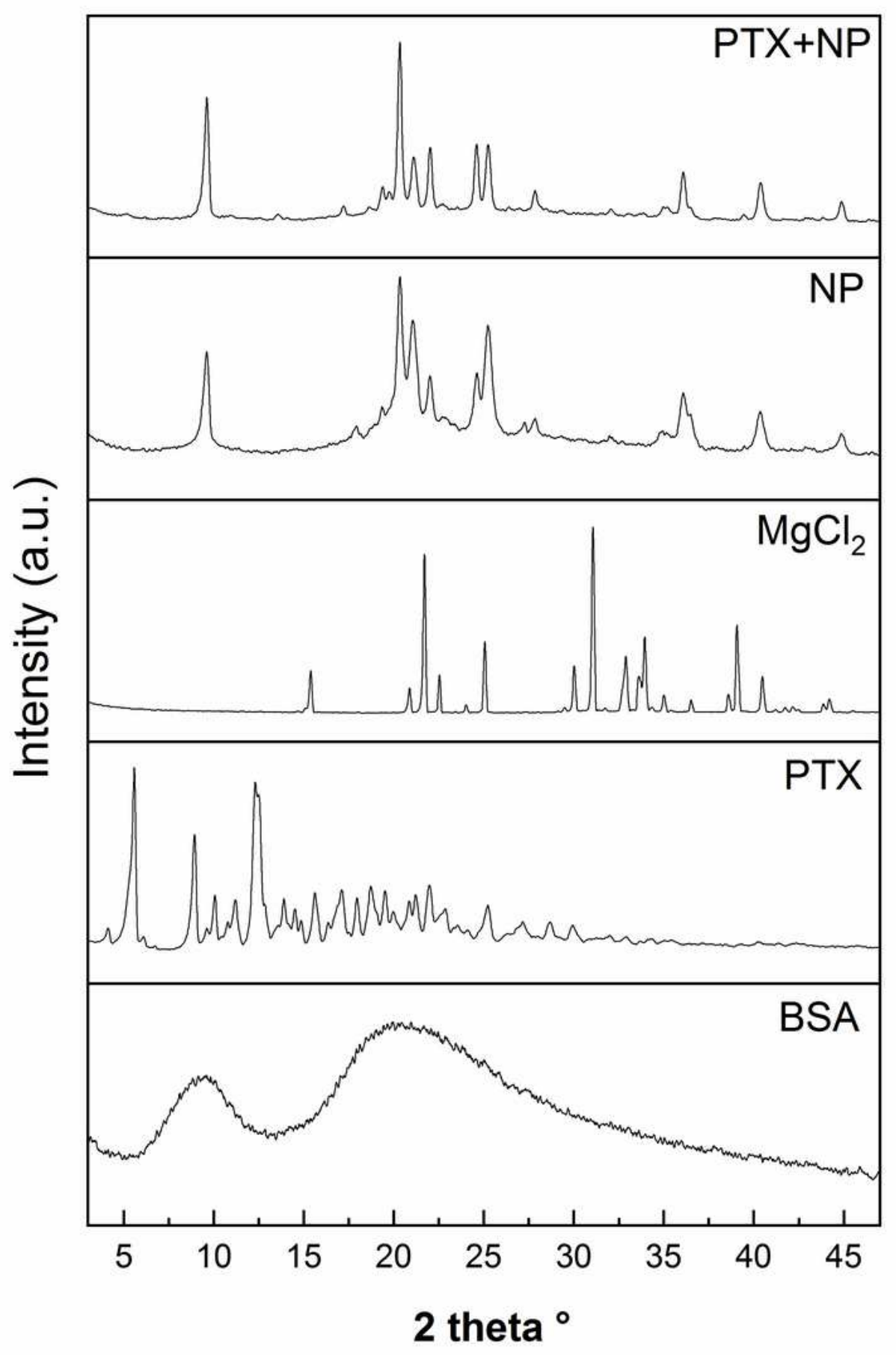

Figure 8

Raman spectrum of BSA; MgCl2; NP; PTX-BSA; and PTX . 


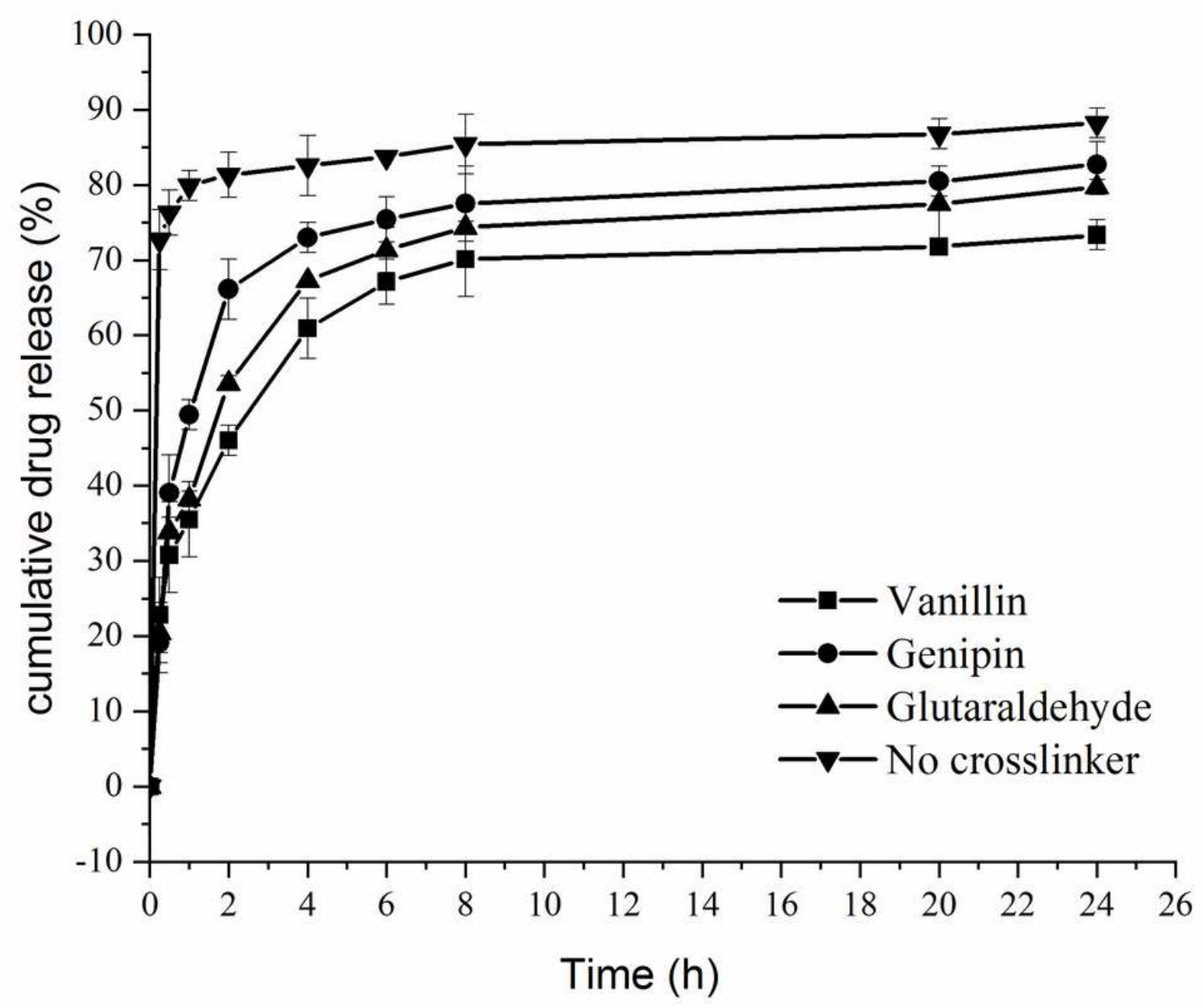

Figure 9

In vitro release profiles of PTX from PTX-BSA. Crosslinking agent dosage, glutaraldehyde/BSA $0.4 \mu \mathrm{l} / \mathrm{mg}$; genipin/BSA $0.05 \mathrm{mg} / \mathrm{mg}$; vanillin/BSA $0.02 \mathrm{mg} / \mathrm{mg}$. 


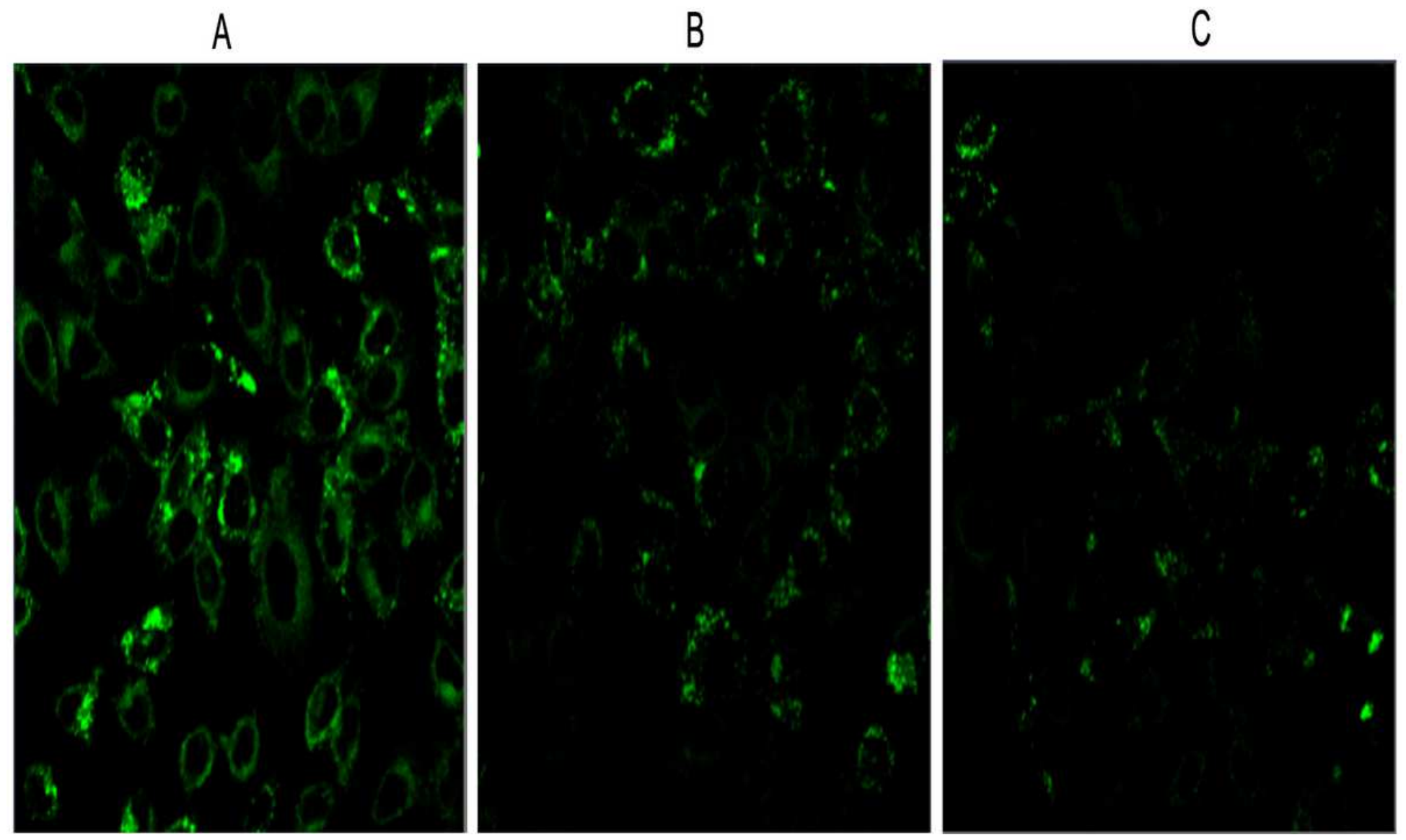

Figure 10

Laser scanning confocal microscope images of HeLa cells after incubation with PTX-BSA. The LSCM images of the FITC-FA-BSA-PTX (A), FITC-BSA-PTX (B) and FITC (C). 